\title{
The Mediating Role of Diabetes Distress and Depressive Symptoms in Type 2 Diabetes Medication Adherence Gender Differences
}

\author{
Benjamin D. Aronson, PharmD, PhD' (i), Kelley J. Sittner, PhD', \\ and Melissa L. Walls, PhD ${ }^{3}$
}

\begin{abstract}
Background. Medication adherence is negatively related to both diabetes distress (DD) and depressive symptoms (DS). Past research suggests gender differences in adherence, DD, and DS. A gap exists in determining if gender differences in adherence are mediated by DD and DS, or if gender moderates differences in adherence by DD/DS. Aims. This study investigated the relationship between gender, DD, DS, and medication adherence and tested for mediating and moderating effects on medication adherence among American Indian adults with type 2 diabetes. Method. The Maawaji idi-oog mino-ayaawin (Gathering for Health) study was a community-based participatory research collaboration with five American Indian tribes. Participants, randomly recruited from clinic records, shared information during computer-assisted personal interviews. This study includes the 166 participants who reported using medications to treat their diabetes. The relationship between gender, DD, DS, and medication adherence are explored. Possible mediating and moderating effects on medication were tested using regression and path analysis. Results. Females had higher levels of DD and DS and lower levels of medication adherence. Higher levels of DD and DS were both associated with lower medication adherence. No evidence was found that gender moderates the relationship between DD or DS and medication adherence. Instead, DD and DS mediated the relationship between gender and medication adherence. Conclusions. Medication adherence differences in male and female patients may be attributable to DD and DS. The present research highlights both DD and DS as targets for clinicians and researchers alike.
\end{abstract}

\section{Keywords}

American Indian, depressive symptoms, diabetes distress, gender, medication adherence, type 2 diabetes

Medication adherence is paramount to diabetes care for those who use medications to manage their disease. Among those with type 2 diabetes, medication adherence is associated with lower hemoglobin A1C (Egede, Gebregziabher, Echols, \& Lynch, 2014; Krapek et al., 2004; Rhee et al., 2005) and is possibly more impactful on A1C than other self-care behaviors (Osborn, Mayberry, \& Kim, 2016). Diabetes medication adherence is associated with decreased emergency department visits, hospitalizations, and related costs (Encinosa, Bernard, \& Dor, 2010), as well as lower rates of diabetes complications (i.e., amputation/ulcers, heart attack, neuropathy, nephrotic syndrome, retinopathy; Gibson et al., 2010). Given the strong link between medication adherence and diabetes control, emergent health care use, costs, and complications, promoting adherence to medications becomes an attractive target for research and intervention.

A significant link between mental health and medication adherence has been illuminated by prior findings. Specifically, both depression and diabetes distress are inversely related to medication adherence. Depression is related to poor medication adherence (Bell et al., 2010; Gellad, Grenard, \& McGlynn, 2009; Gonzalez et al., 2007; Gonzalez et al., 2008; Lin et al., 2004), and depressive symptoms have been implicated as one of four modifiable factors associated with medication adherence (Gellad et al., 2009). Diabetes-related emotional distress is also associated with worse medication adherence (Fisher et al., 2007; Fisher, Glasgow, \& Strycker, 2010; Fisher, Mullan, et al., 2010; Fisher, Skaff et al., 2008). Although related to each other, diabetes distress and depressive symptoms each have distinct, independent relationships with diabetes-related behaviors and health outcomes (Fisher et al., 2007; Fisher, Mullan, et al., 2010;

\footnotetext{
'Ohio Northern University, Ada, OH, USA

${ }^{2}$ Oklahoma State University, Stillwater, OK, USA

${ }^{3}$ Department of International Health, Johns Hopkins Center for American Indian Health, Great Lakes Hub, Duluth, MN, USA
}

\section{Corresponding Author:}

Benjamin D. Aronson, Department of Pharmacy Practice, Raabe College of Pharmacy, Ohio Northern University, 525 S. Main St., Ada, OH 458I0, USA. Email: b-aronson.I@onu.edu 
Fisher, Skaff, et al., 2008; Gonzalez, Kane, Binko, Shapira, \& Hoogendoorn, 2016).

Prior literature has identified possible gender differences in medication adherence. Some studies conclude women are less adherent to chronic medications (Billimek et al., 2015; Chen, Lee, Liang, \& Liao, 2014; Goldstein, Zullig, Bastian, \& Boworth, 2016; Kennedy, Coyne, \& Sclar, 2004; Manteuffel et al., 2014; Zivin, Ratliff, Heisler, Langa, \& Piette, 2010). Other studies have shown that women have greater fear of injections, stigmatization, and reluctance to use insulin (Nam, Chesla, Stotts, Kroon, \& Janson, 2010), and a systematic review showed that across studies female gender was associated with lower adherence to insulin therapy (Davies et al., 2013). However, associations between gender and medication adherence are not robust across studies. Furthermore, women have an increased prevalence of internalizing disorders and diabetes distress. Epidemiologic studies have shown higher rates of internalizing, affective, and mood disorders among women when compared with men (Kessler et al., 1994; Kessler et al., 2005; Kessler, Chiu, Demler, \& Walters, 2005). In a sample of adults with type 2 diabetes, female gender was associated with greater persistence of diabetes distress and depressive affect (Fisher, Skaff, et al., 2008). It may be that higher rates of nonadherence among adult women can be in part attributed to differential rates and longer periods of internalizing problems.

We located only two studies that have explored the intersections of gender, mental health, and medication adherence among those with diabetes. The first specifically investigated the moderating effects of gender on the relationship between depression and adherence to oral diabetes mediations (Nau, Aikens, \& Pacholski, 2007). In this study, women had higher eight-item Patient Health Questionnaire depression scale (PHQ-8) scores, and depression but not gender had a main effect on medication adherence (Nau et al., 2007). Men with depression reported higher nonadherence compared with those without depression, while women with and without depression had relatively comparable levels of adherence (Nau et al., 2007). The second study examined the mediating effects of mental health on the relationship between gender and diabetes adherence among a group of urban youths with type 1 diabetes (Naar-King et al., 2006). In this study, boys had higher rates of externalizing disorders and worse caregiver-reported adherence. The findings from structural equation modeling showed that the relationship between gender and lower adherence was mediated by externalizing symptoms (Naar-King et al., 2006).

Previous research on the intersection of gender, mental health, and diabetes medication adherence is sparse and has important limitations. The first concerns noncomparable ages of the samples (adults vs. adolescents), diverse types of mental health problems measured (internalizing vs. externalizing), and the specific diabetes diagnosis (type 1 vs. type 2), making the results difficult to synthesize. The second concerns the nature of the relationships among mental health, gender, and adherence, specifically whether they involve moderation, mediation, or both. Third, we could locate no studies that assessed the intersection of diabetes distress, gender, and adherence, which is an important oversight due to the salience of diabetes distress for disease management (Fisher et al., 2007; Fisher, Glasgow, et al., 2010; Fisher, Mullan, et al., 2010; Fisher, Skaff, et al., 2008; Gonzalez et al., 2016).

Notably, a majority of studies addressing these issues exclude American Indians (AI), despite the fact that many AI communities experience disproportionate rates of mental distress and type 2 diabetes (Beals et al., 2005; Centers for Disease Control and Prevention, 2017). Furthermore, contexts of medical mistreatment, racism, and historical trauma (Brave Heart \& DeBruyn, 1998; Burnette \& Figley, 2017; Evans-Campbell, 2008; Lewis, Myhra, \& Walker, 2014) may in part fuel lower medication adherence rates for AI diabetes patients (Schmittdiel et al., 2014).

Given the nuanced relationship between depression and diabetes distress (Fisher, Gonzalez, \& Polonsky, 2014) and the limited conflicting evidence surrounding the relationships among gender, mental health, and adherence, it is valuable to further investigate the possible interplay of these factors for medication adherence. The purpose of this study was to (a) investigate the relationship between gender and medication adherence, diabetes distress, and depressive symptoms; (b) test for moderating effects of gender on the relationship between depressive symptoms/diabetes distress and medication adherence; and (c) test for mediation of the relationship between gender and adherence through depressive symptoms and diabetes distress among a sample of AI adults with type 2 diabetes.

\section{Method}

\section{Study Design}

The Maawaji idi-oog mino-ayaawin (Gathering for Health) study is a community-based participatory research project with five AI communities and several university research collaborators. The five communities have provided tribal resolutions in support of the project, and letters of support have been provided by the Indian Health Service (IHS) clinics partnering in this research. Maawaji idi-oog minoayaawin is a mixed-methods study consisting of a qualitative phase followed by a quantitative phase, with further details provided elsewhere (Ratner, Davis, Lhotka, Wille, \& Walls, 2017; Walls, et al., 2017; Elm, Walls, \& Aronson, 2019). The study protocol and procedures were reviewed and approved by the University of Minnesota and Indian Health Service National Institutional Review Boards.

The present research uses the baseline quantitative data from a computer-assisted personal interview (CAPI). Clinic staff at each of the partnering tribal clinics established lists from clinic records of individuals who were 18 years or older 
and had a recently documented diagnosis of type 2 diabetes. Additional inclusion criteria included self-identifying as AI, and living on or proximally near one of the partnering reservations. Clinic staff randomly sampled from these lists, and invited those selected to participate in the quantitative phase of this study. A total of 194 of the 290 eligible and invited individuals provided written consent to participate in the study, a baseline study participation rate of $66.9 \%$. Participants met with trained community interviewers in a location of their choosing to complete a CAPI, and were given $\$ 50$ and a traditional gift of wild rice. The present research includes the 166 participants who reported taking oral or injectable medications to treat their diabetes.

\section{Measures}

The four-item Morisky Medication Adherence Scale (MMAS4) was used as a measure of adherence to diabetes medications (Morisky, Green, \& Levine, 1986). The MMAS-4 asks questions such as "Do you ever forget to take your diabetes medications?" in a yes/no format. "No" responses are summed for a resultant scale ranging from 0 to 4 , where higher scores are indicative of better medication adherence. Only two participants had missing data on Item 2 . The standard procedure for missing data with the MMAS-4 is to impute the median value for that item of those that completed at least $75 \%$ of the items. Thus, the two missing cases were imputed as "No." Cutoff scores for the MMAS-4 are used to categorize individuals as low (score of 0 or 1 ), medium (score of 2 or 3 ), or high (score of 4) adherence. The MMAS-4 correlates well with A1C (Krapek et al., 2004). Cronbach's alpha in this study was .654.

Diabetes-related emotional distress was measured using the Diabetes Distress Screener (DDS-2; Fisher, Glasgow, Mullan, Skaff, \& Polonsky, 2008). Participants were asked on a scale from 1 (not at all bothersome) to 6 (very bothersome), the degree to which two items had distressed or bothered them over the past month. Responses were summed for a resultant scale ranging from 2 to 12 . Cronbach's alpha of the DDS-2 was .643 in this study. Depressive symptoms were measured using the nine-item Patient Health Report (PHQ-9; Kroenke, Spitzer, \& Williams, 2001). Participants provided responses on a scale from 0 (not at all) to 3 (almost every day), regarding the presence of symptoms of depression in the past 2 weeks. These responses are summed with a possible range from 0 to 27, where a higher score indicates higher depressive symptoms. In this study, Cronbach's alpha of the PHQ-9 was .876.

Participants provided their gender as male (0) or female (1). Age at time of interview was calculated from the interview date and date of birth, and recorded in years. Educational attainment was estimated by asking participants the highest level of education completed (i.e., less than high school, high school or GED, some college, vocational or technical training, college graduate, and advanced degree). Per capita annual household income was measured first by assigning the center of the income range selected by a participant and then dividing this household income by the number of individuals reported as living in the household. All participants also were asked if they use insulin to treat their diabetes.

\section{Data Analysis}

SPSS (Version 23) and MPlus (Version 7.1.4; Muthén \& Muthén, 1998-2012) were used for statistical analyses. First, sample statistics were calculated. The bivariate relationships among gender, medication adherence, diabetes distress, and depressive symptoms were examined with independent samples $t$ tests and correlations.

Second, to test for moderating effects of gender on the relationship between depressive symptoms/diabetes distress, we performed multivariate ordinary least squares regression with medication adherence regressed on depressive symptoms, diabetes distress, gender, and two interaction terms (gender $\times$ depressive symptoms, gender $\times$ diabetes distress). Separate models were tested using control variables only, controls and diabetes distress $(+/-$ interaction term), controls and depressive symptoms $(+/-$ interaction term), and controls with diabetes distress and depressive symptoms.

Last, to test for mediation of the relationship between gender and adherence through depressive symptoms and diabetes distress, a path analysis was performed where diabetes distress and depressive symptoms had direct paths to medication adherence, and gender had direct paths to medication adherence, diabetes distress, and depressive symptoms. We controlled for age, educational attainment, per capita annual income, and use of insulin in all multivariate analyses. Although classic tests of mediation (e.g., method recommended by Baron \& Kenny, 1986) often utilize regression analyses, those methods have been the subject of significant debate and the conclusions seem to support the use of more advanced techniques to assess mediation (e.g., Hayes, 2009). Thus, we elected to use path analysis in a structural equation modeling framework to assess whether the relationship between gender and medication adherence could be explained by diabetes distress and/or depressive symptoms.

\section{Results}

Among those taking medications to treat diabetes in the Maawaji idi-oog mino-ayaawin study, the average PHQ-9 score was 5.3 (range 0-27), the average DDS score was 2.56 (range 1-6), and the average MMAS-4 score was 2.54 (range $0-4)$. Based on standard cutoff scores, $20.5 \%$ of participants met criteria for low medication adherence, and $27.7 \%$ for high adherence. The majority were using oral medications only (74.7\%), 6\% using insulin only, and $19.3 \%$ using both oral medications and insulin. Participants ranged in age from 18 to 77 , with a mean age of 45.62 years. The mean per capita household income was $\$ 9,351$. Fifty-three percent of participants identified as female. Mean medication adherence and 
Table I. Medication Adherence (Four-Item Morisky Medication Adherence Scale [MMAS-4]) and Mental Health by Gender.

\begin{tabular}{lccc}
\hline Scale scores & Male & Female & $p^{\mathrm{a}}$ \\
\hline Four-Item Morisky Medication Adherence Scale (mean) & 2.76 & 2.35 & .038 \\
Diabetes Distress Screener (mean) & 2.26 & 2.84 & .006 \\
Patient Health Questionnaire depression scale-9 item (mean) & 4.10 & 6.40 & .010 \\
\hline
\end{tabular}

Note. Use of the MMAS(C) is protected by US copyright laws. Permission for use is required. A Licensure agreement is available from: Donald E. Morisky, ScD, ScM, MSPH, Professor, Department of Community Health Sciences, UCLA School of Public Health, 650 Charles E. Young Drive South, Los Angeles, CA 90095-1772; e-mail: dmorisky@ucla.edu.

andependent-samples $t$ test.

Table 2. Bivariate Correlations Among All Study Variables.

\begin{tabular}{|c|c|c|c|c|c|c|c|c|}
\hline Variable & I & 2 & 3 & 4 & 5 & 6 & 7 & 8 \\
\hline I. Medication adherence & - & & & & & & & \\
\hline 2. Diabetes distress & $-.304 * *$ & - & & & & & & \\
\hline 3. Depressive symptoms & $-.285 * *$ & $.242 * *$ & - & & & & & \\
\hline 4. Gender (female = I) & $-.163^{*}$ & $.211 * *$ & $.207^{* *}$ & - & & & & \\
\hline 5. Age (years) & $.240 * *$ & .019 & -.104 & .046 & - & & & \\
\hline 6. Educational attainment & $.190 *$ & $-.219 * *$ & -.068 & .081 & .110 & - & & \\
\hline 7. Per capita annual income & .144 & $-.189 *$ & $-.177^{*}$ & .015 & $.166^{*}$ & $.340 * *$ & - & \\
\hline 8. On insulin & -.005 & .093 & .033 & -.047 & -.120 & $-.172 *$ & .030 & - \\
\hline
\end{tabular}

$*_{p}<.05 .{ }^{* *} p<.01$.

Table 3. Ordinary Least Squares Regression for Medication Adherence (Four-Item Morisky Medication Adherence Scale [MMAS-4]).

\begin{tabular}{|c|c|c|c|c|c|c|c|c|}
\hline \multirow[b]{2}{*}{ Independent variable } & \multicolumn{2}{|c|}{ Model I } & \multicolumn{2}{|c|}{ Model 2} & \multicolumn{2}{|c|}{ Model 3} & \multicolumn{2}{|c|}{ Model 4} \\
\hline & $\beta$ & $p$ & $\beta$ & $p$ & $\beta$ & $p$ & $\beta$ & $p$ \\
\hline (Constant) & & .022 & & .001 & & .003 & & .000 \\
\hline Gender (female $=\mathrm{I}$ ) & -.19 & .016 & -.13 & .097 & -.14 & .073 & -.10 & .214 \\
\hline Age (years) & .23 & .004 & .24 & .002 & .21 & .007 & .23 & .003 \\
\hline Educational attainment & .17 & .041 & .12 & .130 & .17 & .040 & .13 & .115 \\
\hline Per capita annual income & .05 & .612 & $\ldots .01$ & .873 & .01 & .864 & -.01 & .879 \\
\hline On insulin & .04 & .594 & .07 & .404 & .05 & .517 & .07 & .370 \\
\hline Diabetes distress & & & -.26 & .001 & & & -.23 & .005 \\
\hline Depressive symptoms & & & & & -.22 & .005 & -.18 & .018 \\
\hline Adjusted $R^{2}$ & \multicolumn{2}{|c|}{.095} & \multicolumn{2}{|c|}{.150} & \multicolumn{2}{|c|}{.137} & \multicolumn{2}{|c|}{.176} \\
\hline
\end{tabular}

Note. Bolded text highlights statistically significant coefficients. Use of the MMAS@ is protected by US copyright laws. Permission for use is required. A Licensure agreement is available from: Donald E. Morisky, ScD, ScM, MSPH, Professor, Department of Community Health Sciences, UCLA School of Public Health, 650 Charles E. Young Drive South, Los Angeles, CA 90095-I772; e-mail: dmorisky@ucla.edu.

mental health screener scores are shown for males and females in Table 1. Independent samples $t$ tests indicate that those identifying as female in this study had significantly lower medication adherence $(p=.038)$, higher diabetes distress $(p$ $=.006)$, and higher depressive symptoms $(p=.010)$ than the males.

As shown with bivariate correlations in Table 2, medication adherence was positively associated with age $(r=.240$, $p<.01)$ and educational attainment $(r=.190, p<.05)$, and negatively associated with diabetes distress $(r=-.304, p<$ $.01)$, depressive symptoms $(r=-.285, p<.01)$, and female gender $(r=-.163, p<.05)$. Diabetes distress and depressive symptoms were positively correlated $(r=.242, p$ $<.01)$. Diabetes distress was also positively correlated with female gender $(r=.211, p<.01)$ and negatively correlated with educational attainment $(r=-.219, p<.01)$ and per capita annual income $(r=-.189, p<.05)$. Depressive symptoms were positively correlated with gender $(r=.207$, $p<.01)$ and negatively correlated with per capita annual income $(r=-.177, p<.05)$.

A series of ordinary least squares regression models for medication adherence were tested (Table 3) with gender (Model 1), gender and diabetes distress (Model 2), gender 


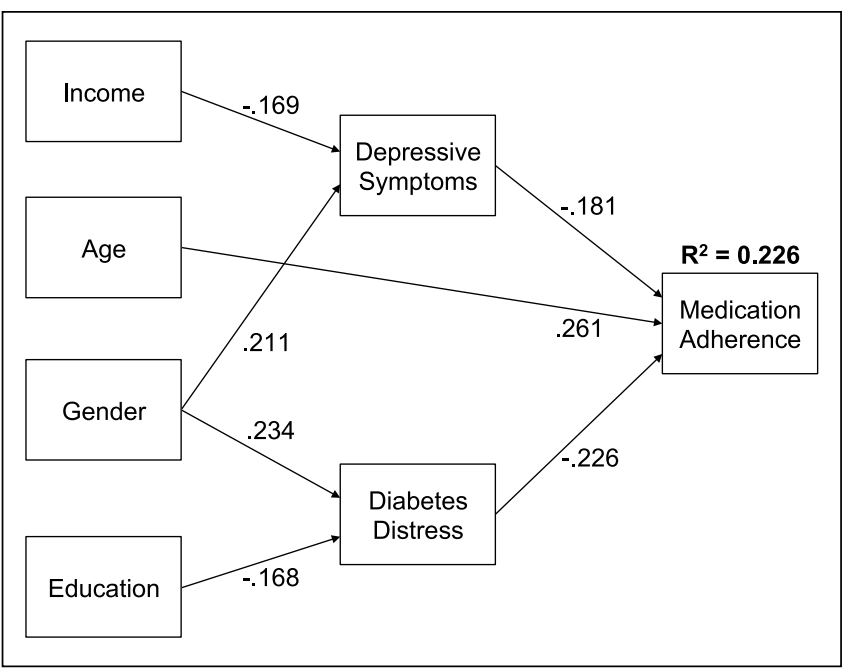

Figure I. Standardized path coefficients from path analysis. *Only significant paths $(p<.05)$ shown. Nonsignificant paths, correlations between variables, and error terms excluded for ease of presentation.

and depressive symptoms (Model 3), and last, gender, diabetes distress, and depressive symptoms (Model 4), net the effects of age, educational attainment, per capita annual income, and use of insulin. Subsequently, possible moderating effects of gender on the relationship between diabetes distress/depressive symptoms and gender were then explored with interaction terms between gender and diabetes distress, and gender and depressive symptoms. In Model 1, female gender $(\beta=-.19, p=.016)$, age $(\beta=.23, p=.004)$, and educational attainment $(\beta=.17, p=.041)$ were related to medication adherence. When either diabetes distress (Model 2) or depressive symptoms was added (Model 3), gender was no longer related to medication adherence. In the fourth model, diabetes distress $(\beta=-.23, p=.005)$ and depressive symptoms $(\beta=-.18, p=.018)$ were each negatively related to medication adherence. Age remained significantly associated with medication adherence $(\beta=.23, p=.003)$. Interaction terms for gender and diabetes distress, and gender and depressive symptoms were nonsignificant (not shown).

Possible mediation of the relationship between gender and medication adherence through depressive symptoms and diabetes distress was explored using path analysis. The path analysis results are shown in Figure 1 and Table 4. Gender had significant indirect and total effects in the model on medication adherence, where female gender was indirectly associated with reduced medication adherence. Nearly half $(48.9 \%)$ of the total effect of gender on medication adherence was explained through diabetes distress (28.5\%) and depressive symptoms (20.4\%). In the path analysis, diabetes distress and depressive symptoms were negatively related to medication adherence, and age was positively related.

\section{Discussion}

This study demonstrates that the relationship between gender and medication adherence among AI adults living with type 2 diabetes appears to be mediated by diabetes distress and depressive symptoms. In other words, the diminished levels of medication adherence seen in this study among women seem to be explained by their higher rates of diabetes distress and depressive symptoms compared with men. The present findings would caution researchers attempting to understand differences in medication adherence by gender; differences may be attenuated if mental health variables are accounted for. Past research including adolescents with type 1 diabetes has declared a similar finding, whereby mental health symptoms mediated the relationship between gender and medication adherence (Naar-King et al., 2006). Naar-King et al. (2006) reported that young girls with type 1 diabetes had higher levels of caregiver reported adherence, which was mediated by lower levels of externalizing symptoms. Dissimilar from the present findings, they did not find an association between depressive symptoms and medication adherence, nor were gender differences in depressive symptoms detected.

In the present study, we move beyond prior literature to investigate the possible mediating role of diabetes distress; we found the positive relationship between diabetes distress and female gender contributes to females' lower medication adherence. This bolsters the recent calls for inclusion of the study of diabetes-related emotional distress when attempting to understand the impact of depression for those with diabetes (Fisher et al., 2014). Contrary to the results of Nau et al. (2007), we did not find evidence of moderation of the relationship between mental health and adherence through gender.

Table 4. Decomposition of Standardized Effects of Gender on Medication Adherence From Path Analysis.

\begin{tabular}{llll}
\hline Variable & Direct effects $[95 \% \mathrm{Cl}]$ & Indirect effects $[95 \% \mathrm{Cl}]$ & Total effects [95\% Cl] \\
\hline Gender & $-.095[-.230, .039]$ & $-.09 I^{* *}[-.160,-.022]$ & $-.186^{* *}[-.320,-.053]$ \\
$\quad$ via Diabetes Distress & & $-.053^{*}[-.103,-.003]$ & $-.038[-.080, .004]$ \\
$\quad$ via Depressive Symptoms & $-.226 *[-.384,-.068]$ & & $-.226 * *[-.384,-.068]$ \\
Diabetes Distress & $-.181 *[-.332,-.030]$ & $-.18 I^{*}[-.332,-.030]$ \\
Depressive Symptoms & & & \\
\hline
\end{tabular}

Note. $\mathrm{Cl}=$ confidence interval.

$*_{p}<.05 . *_{p}<.0$ I. 
The presence of diabetes distress and depressive symptoms was associated with lower medication adherence in this study, consistent with prior findings (Bell et al., 2010; Fisher, Glasgow, et al., 2010; Fisher, Mullan, et al., 2010; Fisher, Skaff, et al., 2008; Gellad et al., 2009; Gonzalez et al., 2007; Gonzalez et al., 2008; Gonzalez et al., 2016; Lin et al., 2004). In our final regression model, we found that diabetes distress and depressive symptoms were both independently associated with lower medication adherence, but the strength of association was higher between diabetes distress and medication adherence than depressive symptoms and medication adherence. Past research has found that diabetes distress is more persistent and better predicts diabetes control than depression (Fisher et al., 2007; Fisher, Glasgow, et al., 2010; Fisher, Mullan, et al., 2010; Fisher, Skaff, et al., 2008). More recently, Gonzalez et al. (2016) found that diabetes distress was associated with lower electronically monitored and self-reported medication adherence, but was not significantly related net the effects of self-reported depression (PHQ-9). Conversely, in a longitudinal study of adults with type 2 diabetes, the association between self-reported depressive symptoms and diet, physical activity, and glycemic control was attenuated net the significant effects of diabetes distress (Fisher et al., 2007). Fisher et al. (2014) recommend considering the context of emotional distress (i.e., stress of managing diabetes, other life stressors) when understanding depressive symptoms in those with diabetes in order to determine the appropriate strategy to ameliorate the emotional distress. Taken as a whole, the present findings paired with past research suggest that measuring both depression and diabetes distress are important, as is understanding the patient context for current symptoms.

Clinicians caring for those with type 2 diabetes can use these results to improve care. A large literature has shown the importance of medication adherence among those with diabetes (Egede et al., 2014; Encinosa et al., 2010; Gibson et al., 2010; Krapek et al., 2004; Osborn et al., 2016; Rhee et al., 2005). Interventions to care for possible mental health issues may have positive impacts on medication adherence, which would be hypothesized to improve disease control, and decrease downstream consequences and health care spending. Given that the nation spends $\$ 237$ billion in direct costs for diabetes (American Diabetes Association, 2018b), and the IHS spends 37\% of their health care dollars on diabetes costs (O'Connell, Wilson, Manson, \& Acton, 2012), any interventions to improve medication adherence may pay substantial dividends. The current American Diabetes Association (2018a) guidelines recommend that providers screen and routinely monitor diabetes distress, as well as screening for depression and other psychosocial problems and barriers to diabetes self-management.

\section{Limitations}

Of importance are some limitations in this work. The relationships modeled in the path analysis are not causal given the cross-sectional nature of the data. The measure of medication adherence used in this study represents a validated approximation, but ignores possible culturally specific interpretations and meanings of medications. For instance, some community members may view prescription medications as a symbol of historical trauma and colonization, given the context of U.S. government-provided health care. We acknowledge past historical atrocities perpetrated by the U.S. government and the legacy of medical racism (Brave Heart \& DeBruyn, 1998; Burnette \& Figley, 2017; Evans-Campbell, 2008; Lewis et al., 2014) that help us understand justifiable mistrust and historically rooted causes of not using medications or using medications in a manner which could be deemed as "nonadherent." In addition, others have noted limitations of the MMAS-4, such as potentially missing reasons for nonadherence, and instances of inconsistent relationship with clinical outcomes (Tan, Patel, \& Chang, 2014). The MMAS-4 was chosen as it best fit the present study design (Clifford, Perez-Nieves, Skalicky, Reaney, \& Coyne, 2014), but these limitations should be considered when interpreting our findings.

Of note, other clinical variables could be used in tandem with a self-reported measure of medication adherence. For instance, glycemic control (e.g., fasting blood sugar or A1c) or complications (e.g., micro- and macrovascular comorbidities) could also signal poor medication adherence; however, this assumes all medication regimens have been optimized to ensure disease control. These variables were not included in this study due to issues of temporal ordering: past glycemic control and complications collected from clinic records would not provide an accurate indicator of current medication adherence.

There has been considerable attention to gender differences in reporting of internalizing symptoms, with some authors arguing that men underreport such symptoms (Smith, Mouzon, \& Elliott, 2018), and others demonstrating that women actually experience more mental distress than men (Mirowsky \& Ross, 1995). Further investigation is needed to consider these issues in general and in Indigenous communities in particular.

General aversion to injecting medications could potentially impact medication adherence for those who use insulin, yet we did not control for insulin use in the path analysis. Use of insulin in this study was not associated with medication adherence; thus, we decided against including this control variable, opting for a more parsimonious path analysis model.

Finally, there are currently 573 federally recognized AI and Alaska Native tribes in the United States, and many more not recognized. As with all racial and ethnic groups, there is considerable diversity within and between communities. These differences may affect the generalizability of this work to other Native and non-Native communities.

\section{Implications for Practice}

In this study of diabetes among AI adults, women had both lower levels of medication adherence and higher levels of 
diabetes distress and depressive symptoms. No evidence was found that the relationships between diabetes distress and medication adherence, or between depressive symptoms and adherence are moderated by gender. Instead, the results suggest the relationship between gender and medication adherence is mediated through diabetes distress and depressive symptoms. Diabetes distress in particular has a strong relationship with medication adherence and should be evaluated and addressed by clinicians.

\section{Acknowledgments}

Sidnee Kellar, Rose Barber, Robert Miller, Tweed Shuman, Lorraine Smith, Sandy Zeznanski, Patty Subera, Tracy Martin, Geraldine Whiteman, Lisa Perry, Trisha Prentice, Alexis Mason, Charity Prentice-Pemberton, Kathy Dudley, Mona Nelson, Eileen Miller, Geraldine Brun, Murphy Thomas, Mary Sikora-Petersen, Tina Handeland, GayeAnn Allen, Frances Whitfield, Phillip Chapman Sr., Hope Williams, Betty Jo Graveen, Daniel Chapman Jr., Sonya Psuik, Doris Isham, Stan Day, Jane Villebrun, Beverly Steel, Muriel Deegan, Peggy Connor, Michael Connor, Ray E. Villebrun Sr., Pam Hughes, Cindy McDougall, Melanie McMichael, Robert Thompson, and Sandra Kier.

\section{Declaration of Conflicting Interests}

The authors declared no potential conflicts of interest with respect to the research, authorship, and/or publication of this article.

\section{Funding}

The authors disclosed receipt of the following financial support for the research, authorship, and/or publication of this article: Research reported in this article was supported by the National Institute of Diabetes and Digestive and Kidney Diseases of the National Institutes of Health (DK091250, M. Walls, PI). The content is solely the responsibility of the authors and does not necessarily represent the official views of the National Institutes of Health. B. Aronson gratefully acknowledges support from a Health Services Dissertation Award (R36) from the Agency for Healthcare Research and Quality (1R36HS024180-01).

\section{ORCID iD}

Benjamin D. Aronson (iD https://orcid.org/0000-0001-9739-6221

\section{References}

American Diabetes Association. (2018a). Comprehensive medical evaluation and assessment of comorbidities. Sec. 3. Standards of Medical Care in Diabetes-2018. Diabetes Care, 41(Suppl. 1), S28-S50. doi:10.2337/dc18-S003

American Diabetes Association. (2018b). Economic costs of diabetes in the US in 2017. Diabetes Care, 41,917-928. doi:10.2337/ dci18-0007

Baron, R. M., \& Kenny, D. A. (1986). The moderator-mediator variable distinction in social psychological research: Conceptual, strategic, and statistical considerations. Journal of Personality and Social Psychology, 51, 1173-1182.

Beals, J., Novins, D. K., Whitesell, N. R., Spicer, P., Mitchell, C. M., \& Manson, S. M. (2005). Prevalence of mental disorders and utilization of mental health services in two American Indian reservation populations: Mental health disparities in a national context. American Journal of Psychiatry, 162, 17231732. doi:10.1176/appi.ajp.162.9.1723

Bell, R. A., Andrews, J. S., Arcury, T. A., Snively, B. M., Golden, S. L., \& Quandt, S. A. (2010) Depressive symptoms and diabetes self-management among rural older adults. American Journal of Health Behavior, 34, 36-44. doi:10.5993/AJHB.34.1.5

Billimek, J., Malik, S., Sorkin, D. H., Schmalbach, P., Ngo-Metzger, Q., Greenfield, S., \& Kaplan, S. H. (2015). Understanding disparities in lipid management among patients with type 2 diabetes: Gender differences in medication nonadherence after treatment intensification. Women's Health Issues, 25(1), 6-12. doi:10.1016/j.whi.2014.09.00

Brave Heart, M. Y. H., \& DeBruyn, L. M. (1998). The American Indian holocaust: Healing historical unresolved grief. American Indian and Alaska Native Mental Health Research, 8(2), 60-82.

Burnette, C. E., \& Figley, C. R. (2017). Historical oppression, resilience, and transcendence: Can a holistic framework help explain violence experienced by indigenous people? Social Work, 62(1), 37-44. doi:10.1093/sw/sww065

Centers for Disease Control and Prevention. (2017). Summary health statistics: National Health Interview Survey: 2015. Atlanta, GA: Author.

Chen, S. L., Lee, W. L., Liang, T., \& Liao, I. C. (2014). Factors associated with gender differences in medication adherence: A longitudinal study. Journal of Advanced Nursing, 70, 20312040. doi:10.1111/jan.12361

Clifford, S., Perez-Nieves, M., Skalicky, A. M., Reaney, M., \& Coyne, K. S. (2014). A systematic literature review of methodologies used to assess medication adherence in patients with diabetes. Current Medical Research and Opinion, 30, 1071-1085.

Davies, M. J., Gagliardino, J. J., Gray, L. J., Khunti, K., Mohan, V., \& Hughes, R. (2013). Real-world factors affecting adherence to insulin therapy in patients with type 1 or type 2 diabetes mellitus: A systematic review. Diabetic Medicine, 30, 512-524. doi: $10.1111 /$ dme. 12128

Egede, L. E., Gebregziabher, M., Echols, C., \& Lynch, C. P. (2014). Longitudinal effects of medication nonadherence on glycemic control. Annals of Pharmacotherapy, 48, 562-570. doi: $10.1177 / 1060028014526362$

Elm, J. H. L., Walls, M. L., \& Aronson, B. D. (2019). Sources of stress among Midwest American Indian adults with type 2 diabetes. American Indian and Alaska Native Mental Health Research, 26, 33-62. doi:10.5820/aian.2601.2019.33

Encinosa, W. E., Bernard, D., \& Dor, A. (2010). Does prescription drug adherence reduce hospitalizations and cost? The case of diabetes. Advances in Health Economics and Health Services Research, 22, 151-173. doi:10.1108/S0731-2199(2010)0000022010

Evans-Campbell, T. (2008). Historical trauma in American Indian/Native Alaska communities: A multilevel framework for exploring impacts on individuals, families, and communities. Journal of Interpersonal Violence, 23, 316-338. doi:10.1177/0886260507312290

Fisher, L., Glasgow, R. E., Mullan, J. T., Skaff, M. M., \& Polonsky, W. H. (2008). Development of a brief diabetes distress screening instrument. Annals of Family Medicine, 6, 246-252. doi: $10.1370 / \mathrm{afm} .842$

Fisher, L., Glasgow, R. E., \& Strycker, L.A. (2010). The relationship between diabetes distress and clinical depression with glycemic 
control among patients with type 2 diabetes. Diabetes Care, 33, 1034-1036. doi:10.2337/dc09-2175

Fisher, L., Gonzalez, J. S., \& Polonsky, W. H. (2014). The confusing tale of depression and distress in patients with diabetes: A call for greater clarity and precision. Diabetic Medicine, 31, 764772. doi:10.2337/dc09-2175

Fisher, L., Mullan, J. T., Arean, P., Glasgow, R. E., Hessler, D., \& Masharani, U. (2010). Diabetes distress but not clinical depression or depressive symptoms is associated with glycemic control in both cross-sectional and longitudinal analyses. Diabetes Care, 33, 23-28. doi:10.2337/dc09-1238

Fisher, L., Skaff, M. M., Mullan, J. T., Arean, P., Glasgow, R., \& Masharani, U. (2008). A longitudinal study of affective and anxiety disorders, depressive affect and diabetes distress in adults with type 2 diabetes. Diabetic Medicine, 25, 1096-1101. doi:10.1111/j.1464-5491.2008.02533.x

Fisher, L., Skaff, M. M., Mullan, J. T., Arean, P., Mohr, D., Masharani, U., . . L Laurencin, G. (2007). Clinical depression versus distress among patients with type 2 diabetes: Not just a question of semantics. Diabetes Care, 30, 542-548. doi: $10.2337 / \mathrm{dc} 06-1614$

Gellad, W. F., Grenard, J., \& McGlynn, E. A. (2009). A review of barriers to medication adherence: A framework for driving policy options. Santa Monica, CA: RAND.

Gibson, T. B., Song, X., Alemayehu, B., Wang, S. S., Waddell, J. L., Bouchard, J. R., \& Forma, F. (2010). Cost sharing, adherence, and health outcomes in patients with diabetes. American Journal of Managed Care, 16, 589-600.

Goldstein, K. M., Zullig, L. L., Bastian, L. A., \& Boworth, H. B. (2016). Statin adherence: Does gender matter? Current Atherosclerosis Reports, 18(11), 63. doi:10.1007/s11883-016-0619-9

Gonzalez, J. S., Kane, N. S., Binko, D. H., Shapira, A., \& Hoogendoorn, C. J. (2016). Tangled up in blue: Unraveling the links between emotional distress and treatment adherence in type 2 diabetes. Diabetes Care, 39, 2182-2189. doi:10.2337/ dc16-1657

Gonzalez, J. S., Peyrot, M., McCarl, L. A., Collins, E. M., Serpa, L., Mimiaga, M. J., \& Safren, S. A. (2008). Depression and diabetes treatment nonadherence: A meta-analysis. Diabetes Care, 31, 2398-2403. doi:10.2337/dc08-1341

Gonzalez, J. S., Safren, S. A., Cagliero, E., Wexler, D. J., Delahanty, L., Wittenberg, E., . . . Grant, R. W. (2007). Depression, selfcare, and medication adherence in type 2 diabetes: Relationships across the full range of symptom severity. Diabetes Care, 30, 2222-2227. doi: $10.2337 / \mathrm{dc} 07-0158$

Hayes, A. F. (2009). Beyond Baron and Kenny: Statistical mediation analysis in the new millennium. Communication Monographs, 76, 408-420. doi:10.1080/03637750903310360

Kennedy, J., Coyne, J., \& Sclar, D. (2004). Drug affordability and prescription noncompliance in the United States: 19972002. Clinical Therapeutics, 26, 607-614. doi:10.1016/S01492918(04)90063-X

Kessler, R. C., Berglund, P., Demler, O., Jin, R., Merikangas, K. R., $\&$ Walters, E. E. (2005). Lifetime prevalence and age-of-onset distributions of DSM-IV disorders in the National Comorbidity Survey Replication. Archives of General Psychiatry, 62, 593602. doi:10.1001/archpsyc.62.6.593

Kessler, R. C., Chiu, W. T., Demler, O., \& Walters, E. E. (2005). Prevalence, severity, and comorbidity of 12-month DSM-IV disorders in the National Comorbidity Survey Replication. Archives of General Psychiatry, 62, 617-627. doi:10.1001/ archpsyc.62.6.617

Kessler, R. C., McGonagle, K. A., Zhao, S., Nelson, C. B., Hughes, M., Eshleman, S., . . .Kendler, K. S. (1994). Lifetime and 12-month prevalence of DSM-III-R psychiatric disorders in the United States: Results from the National Comorbidity Survey. Archives of General Psychiatry, 51, 8-19. doi:10.1001/archpsyc. 1994.03950010008002

Krapek, K., King, K., Warren, S. S., George, K. G., Caputo, D. A., Mihelich, K., ... W Walden, S. (2004). Medication adherence and associated hemoglobin A1c in type 2 diabetes. Annals of Pharmacotherapy, 38, 1357-1362. doi:10.1345/aph.1D612

Kroenke, K., Spitzer, R. L., \& Williams, J. B. (2001). The PHQ-9: Validity of a Brief Depression Severity Measure. Journal of General Internal Medicine, 16, 606-613. doi:10.1046/j.15251497.2001.016009606.x

Lewis, M., Myhra, L., \& Walker, M. (2014). Advancing health equity in medical family therapy research. In J. Hodgson, A. Lamson, T. Mendenhall, \& D. R. Crane (Eds.), Medical family therapy: Advanced applications (pp. 319-340). Cham, Switzerland: Springer International.

Lin, E. H., Katon, W., Von Korff, M., Rutter, C., Simon, G. E., Oliver, M., . . . Young, B. (2004). Relationship of depression and diabetes self-care, medication adherence, and preventive care. Diabetes Care, 27, 2154-2160. doi:10.2337/diacare.27.9.2154

Manteuffel, M., Williams, S., Chen, W., Verbrugge, R. R., Pittman, D. G., \& Steinkellner, A. (2014). Influence of patient sex and gender on medication use, adherence, and prescribing alignment with guidelines. Journal of Women's Health, 23, 112119. doi:10.1089/jwh.2012.3972

Mirowsky, J., \& Ross, C. E. (1995). Sex differences in distress: Real or artifact? American Sociological Review, 60, 449-468.

Morisky, D. E., Green, L. W., \& Levine, D. M. (1986). Concurrent and predictive validity of a self-reported measure of medication adherence. Medical Care, 24, 67-74.

Muthén, L. K., \& Muthén, B. O. (1998-2012). Mplus user's guide (7th ed.). Los Angeles, CA: Muthén \& Muthén.

Naar-King, S., Idalski, A., Ellis, D., Frey, M., Templin, T., Cunningham, P. B., \& Cakan, N. (2006). Gender differences in adherence and metabolic control in urban youth with poorly controlled type 1 diabetes: The mediating role of mental health symptoms. Journal of Pediatric Psychology, 31, 793-802. doi:10.1093/jpepsy/jsj090

Nam, S., Chesla, C., Stotts, N. A., Kroon, L., \& Janson, S. L. (2010). Factors associated with psychological insulin resistance in individuals with type 2 diabetes. Diabetes Care, 33, 1747-179. doi:10.2337/dc10-0099

Nau, D. P., Aikens, J. E., \& Pacholski, A. M. (2007). Effects of gender and depression on oral medication adherence in persons with type 2 diabetes mellitus. Gender Medicine, 4, 205-213. doi:10.1016/S1550-8579(07)80041-6

O'Connell, J. M., Wilson, C., Manson, S. M., \& Acton, K. J. (2012). The costs of treating American Indian adults with diabetes within the Indian Health Service. American Journal of Public Health, 102, 301-308. doi:10.2105/AJPH.2011.300332

Osborn, C. Y., Mayberry, L. S., \& Kim, J. M. (2016). Medication adherence may be more important than other behaviours for 
optimizing glycaemic control among low-income adults. Journal of Clinical Pharmacology and Therapeutics, 41, 256-259. doi:10.1111/jcpt.12360

Ratner, N. L., Davis, E. B., Lhotka, L. L., Wille, S. M., \& Walls, M. L. (2017). Patient-centered care, diabetes empowerment, and type 2 diabetes medication adherence among American Indian patients. Clinical Diabetes, 35, 281-285.

Rhee, M. K., Slocum, W., Ziemer, D. C., Culler, S. D., Cook, C. B., El-Kebbi, I. M., . . Phillips, L. S. (2005). Patient adherence improves glycemic control. Diabetes Educator, 31, 240-250. doi: $10.1177 / 0145721705274927$

Schmittdiel, J. A., Steiner, J. F., Adams, A. S., Dyer, W., Beals, J., Henderson, W. G., . . . Waitzfelder, B. (2014). Diabetes care and outcomes for American Indians and Alaska Natives in commercial integrated delivery systems: A SUrveillance, PREvention, and ManagEment of diabetes mellitus (SUPREME-DM) study. BMJ Open Diabetes Research \& Care, 2(1), e000043. doi:10.1136/bmjdrc-2014-000043
Smith, D. T., Mouzon, D. M., \& Elliott, M. (2018). Reviewing the assumptions about men's mental health: An exploration of the gender binary. American Journal of Men's Health, $12,78-89$.

Tan, X., Patel, I., \& Chang, J. (2014). Review of the four item Morisky Medication Adherence Scale (MMAS-4) and eight item Morisky Medication Adherence Scale (MMAS-8). Innovations in Pharmacy, 5(3), 165. doi:10.24926/iip.v5i3 .347

Walls, M. L., Sittner, K. J., Aronson, B. D., Forsberg, A. K., Whitbeck, L. B., \& Al'absi, M. (2017). Stress exposure and physical, mental, and behavioral health among American Indian adults with type 2 diabetes. International Journal of Environmental Research and Public Health, 14, 1074. doi:10.3390/ijerph14091074

Zivin, Z., Ratliff, S., Heisler, M. M., Langa, K. M., \& Piette, J. D. (2010). Factors influencing cost-related nonadherence to medication in older adults: A conceptually based approach. Value in Health, 13, 338-345. doi:10.1111/j.1524-4733.2009.00679.x 